\title{
Effects of Solid Precipitation and Surface Corrosion on the Adhesion Strengths of Sintered Hydrate Deposits on Pipe Walls
}

Chenwei Liu $^{a, b *}$, Xu Zeng ${ }^{b}$, Ci Yan ${ }^{b}$, Chenru Zhou ${ }^{b}$, Mingzhong Li ${ }^{a, b}$, Zhiyuan Wang ${ }^{a, b *}$

${ }^{a}$ Key Laboratory of Unconventional Oil \& Gas Development (China University of Petroleum (East China)), Ministry of Education, Qingdao 266580, P. R. China

${ }^{b}$ School of Petroleum Engineering, China University of Petroleum (East China), Qingdao 266580, P. R. China

\section{Corresponding Authors}

*E-mail: Wangzy1209@126.com

liuchenwei_upc@hotmail.com

\section{Contents}

Figure. S1 Photographs of custom-built hydrate adhesion strength measurement system...............S2

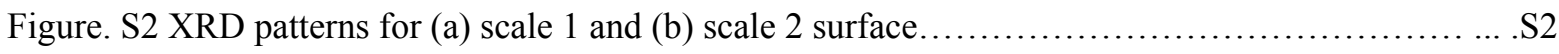

Figure. S3 The SEM of the steel substrate polished with (a) 80 mesh and (b) 1000 mesh sandpapers...... S3 


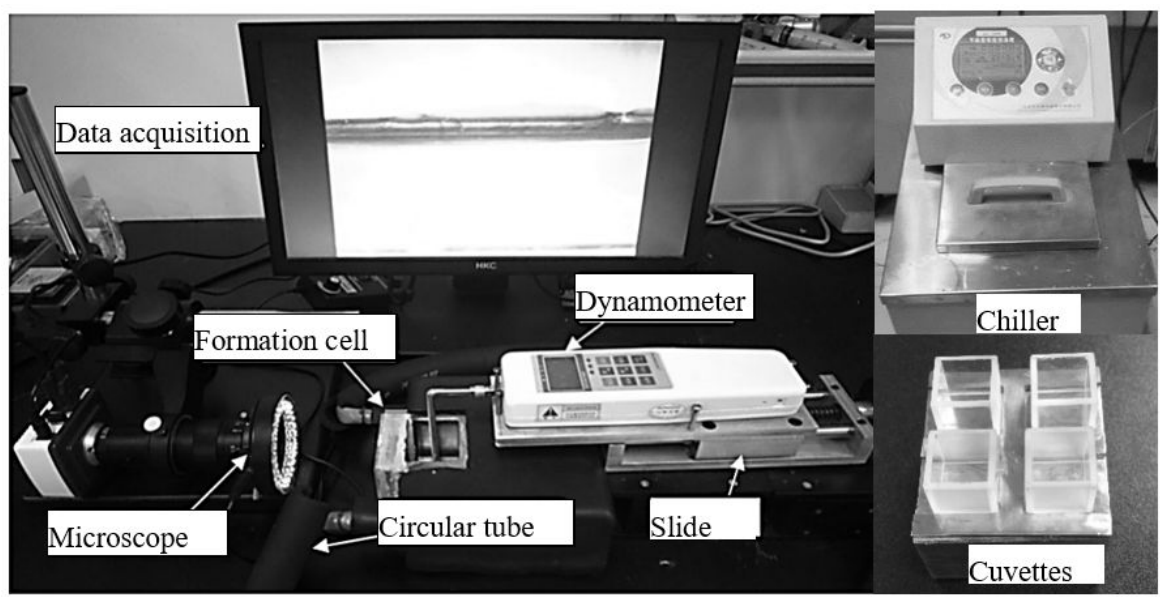

Figure. S1 Photographs of custom-built hydrate adhesion strength measurement system

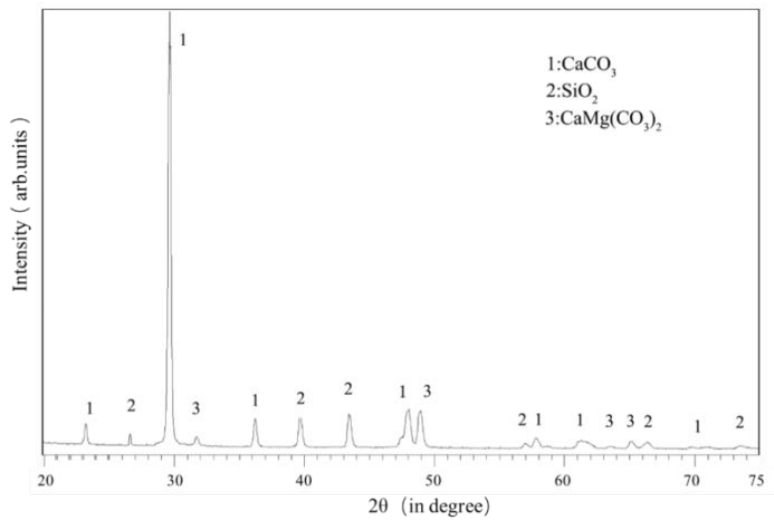

(a)

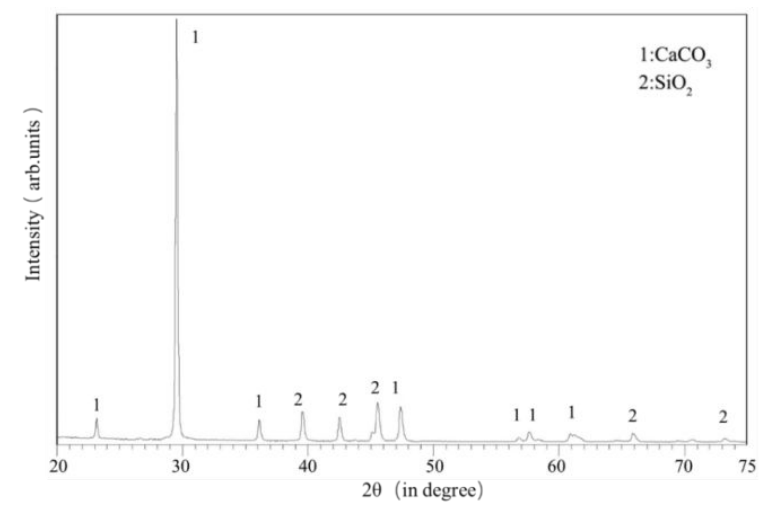

(b)

Figure.S2 XRD patterns for (a) scale 1 and (b) scale 2 surface 


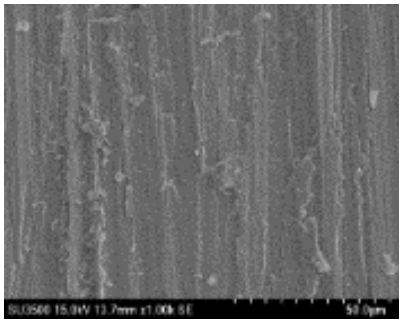

(a)

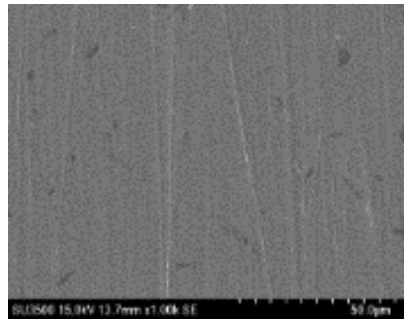

(b)

Figure. S3 The SEM of the steel substrate polished with (a) 80mesh and (b) 1000mesh sandpapers 